\title{
Factors Associated with Gender-Affirming Surgery and Age of Hormone Therapy Initiation Among Transgender Adults
}

\author{
Noor Beckwith, ${ }^{1,2}$ Sari L. Reisner, ${ }^{2-5}$ Shayne Zaslow, ${ }^{3,6}$ Kenneth H. Mayer, ${ }^{2,3,7}$ and Alex S. Keuroghlian ${ }^{1-3, *}$
}

\begin{abstract}
Purpose: Gender-affirming surgeries and hormone therapy are medically necessary treatments to alleviate gender dysphoria; however, significant gaps exist in the research and clinical literature on surgery utilization and age of hormone therapy initiation among transgender adults.

Methods: We conducted a retrospective review of electronic health record data from a random sample of 201 transgender patients of ages 18-64 years who presented for primary care between July 1, 2010 and June 30, 2015 (inclusive) at an urban community health center in Boston, MA. Fifty percent in our analyses were trans masculine (TM), 50\% trans feminine, and 24\% reported a genderqueer/nonbinary gender identity. Regression models were fit to assess demographic, gender identity-related, sexual history, and mental health correlates of genderaffirming surgery and of age of hormone therapy initiation.

Results: Overall, $95 \%$ of patients were prescribed hormones by their primary care provider, and the mean age of initiation of masculinizing or feminizing hormone prescriptions was 31.8 years $(S D=11.1)$. Younger age of initiation of hormone prescriptions was associated with being TM, being a student, identifying as straight/heterosexual, having casual sexual partners, and not having past alcohol use disorder. Approximately one-third (32\%) had a documented history of gender-affirming surgery. Factors associated with increased odds of surgery were older age, higher income levels, not identifying as bisexual, and not having a current psychotherapist.

Conclusion: This study extends our understanding of prevalence and factors associated with gender-affirming treatments among transgender adults seeking primary care. Findings can inform future interventions to expand delivery of clinical care for transgender patients.
\end{abstract}

Keywords: barriers to care; gender-affirming hormone therapy; gender-affirming surgery; mental health; sexual health; transgender

\section{Introduction}

Gender-affirming hormone therapy and surgeries ${ }^{1,2}$ are deemed medically necessary treatments for gender dysphoria by the American Medical Association and other clinical policy-setting organizations. ${ }^{3}$ Access to gender-affirming hormone therapy and surgeries is as- sociated with improvements in psychological functioning and quality of life among transgender adults, ${ }^{4-6}$ and decreased risk of suicidal ideation and substance use disorders. ${ }^{7,8}$ Nevertheless, transgender adults continue to experience numerous barriers to accessing and receiving gender-affirming medical and surgical care,

\footnotetext{
'Department of Psychiatry, Massachusetts General Hospital, Boston, Massachusetts.

${ }^{2}$ Harvard Medical School, Boston, Massachusetts.

${ }^{3}$ Division of Education and Training, The Fenway Institute, Fenway Health, Boston, Massachusetts.

${ }^{4}$ Division of General Pediatrics, Boston Children's Hospital, Boston, Massachusetts.

${ }^{5}$ Department of Epidemiology, Harvard T.H. Chan School of Public Health, Boston, Massachusetts.

${ }^{6}$ Department of Sociology, University of Virginia, Charlottesville, Virginia.

${ }^{7}$ Department of Medicine, Beth Israel Deaconess Medical Center, Boston, Massachusetts.
}

*Address correspondence to: Alex S. Keuroghlian, MD, MPH, Division of Education and Training, The Fenway Institute, Fenway Health, 1340 Boylston Street, Boston, MA 02215, E-mail: akeuroghlian@partners.org

(c) Noor Beckwith et al. 2017; Published by Mary Ann Liebert, Inc. This is an Open Access article distributed under the terms of the Creative Commons Attribution License, which permits unrestricted use, distribution, and reproduction in any medium, provided the original work is properly cited. 
including harassment and denial of treatment by providers, fear of mistreatment based on their gender identity resulting in not seeking needed healthcare, lack of financial resources needed to access care, and ongoing denial of insurance coverage for gender-affirming hormone therapy and surgical procedures. ${ }^{9,10}$ Health professionals typically receive minimal training in core clinical competencies related to transgender health, and healthcare settings often fail to provide inclusive, affirming environments for transgender patients. ${ }^{11}$ Because of barriers to accessing gender-affirming medical and surgical care, transgender people are often relegated to unsafe medically unmonitored hormone use and other body modifications (e.g., unmonitored silicone injections) to affirm their gender and alleviate distress. ${ }^{12-18}$

Little is known about factors associated with genderaffirming surgery and age of initiation of hormone therapy among transgender adults. Transgender adults vary significantly in their access to and selection of possible hormone therapy, surgical procedures, or both to affirm their gender through personalized care plans. $^{2,19-21}$ Transgender people also vary across the lifespan with regard to the age at which they initiate gender-affirming medical care, ${ }^{2}$ and factors influencing individual variability in the age of hormone initiation are poorly understood. Existing research on barriers to transgender healthcare access has relied largely on participant self-report rather than direct methods such as medical documentation. ${ }^{10}$ One such study using self-report identified treatment costs and lack of qualified providers as barriers to optimal care, and found that transgender respondents $>50$ years old and those in committed relationships were less likely to report plans for future gender-affirming hormone therapy. ${ }^{22}$

There remain significant gaps in the literature regarding the relationship between demographics, gender identity, sexual history, and mental health to hormone therapy initiation and surgery utilization among transgender adults. To address these gaps, this study used a retrospective electronic health record (EHR) review to (1) assess the prevalence and distribution of gender-affirming hormone therapy and surgeries among transgender adults at a Boston community health center with specialized care for sexual and gender minority populations and (2) examine the association of gender-affirming surgery utilization and age of hormone therapy initiation with demographics, gender identity, sexual history, and mental health in this traditionally underserved population.

\section{Methods}

Study participants and procedures

We conducted a retrospective review of EHR data from a random sample of 201 transgender patients of ages 18 64 years who presented for one or more healthcare visit(s) between July 1, 2010 and June 30, 2015 (inclusive) at an urban community health center in Boston, MA, specializing in LGBT (lesbian, gay, bisexual, and transgender) healthcare. ${ }^{23,24}$ This study period was the 5year time frame immediately before beginning the EHR review process. Patients were identified as transgender by automated query based on a standardized flag system in the EHR used to designate all transgenderidentified patients at the health center. The sample was drawn from the pool of all 1683 transgender-identified patients of 18 years or older who had presented for care within the 5-year study period, using an automated simple random sampling algorithm. ${ }^{25}$ Data were treated as a cross-sectional sample. Variables related to demographics, gender identity, sexual history, and mental health were extracted through a combination of automated query and manual audit methods from the EHR. Variables extracted by automated query were systematically confirmed by manual audit of EHR visit notes. Variables of interest were operationally defined with specific parameters and collected through prespecified systematic protocols to minimize potential bias related to recall, missing data, and other factors during the EHR data extraction process. ${ }^{26,27}$ The study was approved by the health center's Institutional Review Board.

\section{Variables and operationalization}

Outcomes. The two outcome variables in this study were (1) history of any gender-affirming surgery (yes, no) and (2) age of gender-affirming hormone therapy initiation (years). We determined age of hormone therapy initiation for each participant by manual EHR audit to assess the date of first hormone therapy, which was systematically documented for transgender patients in the health center's EHR. History of any gender-affirming surgery (chest construction, breast augmentation, facial feminization, phalloplasty, metoidioplasty, vaginoplasty, hysterectomy, oophorectomy, orchiectomy, or other gender-affirming procedures) was determined by manual EHR audit, as these surgical procedures were also systematically documented in the EHR.

Statistical predictors. We assessed statistical predictors of age of hormone therapy initiation and of any genderaffirming surgery in four areas: (1) demographics, (2) 
Table 1. Demographics

\begin{tabular}{|c|c|c|c|c|}
\hline Variable & $\begin{array}{c}\text { TM } \\
(n=73)\end{array}$ & $\begin{array}{c}\mathrm{TF} \\
(n=72)\end{array}$ & $\begin{array}{c}\text { Total } \\
(N=145)\end{array}$ & $\begin{array}{l}\text { TM vs. } \\
\text { TF, } p\end{array}$ \\
\hline \multicolumn{5}{|l|}{ Age in years } \\
\hline Mean (SD) & $27.9(6.9)$ & 35.7 (13.7) & $31.8(11.5)$ & \multirow{3}{*}{0.001} \\
\hline Median & 25.0 & 30.0 & 27.0 & \\
\hline Range & $19-50$ & $21-64$ & $19-64$ & \\
\hline \multicolumn{5}{|c|}{ Population age strata in sample, $n(\%)$} \\
\hline $18-25$ years & $40(54.8)$ & $24(33.3)$ & $64(44.1)$ & \multirow[t]{3}{*}{$<0.001$} \\
\hline $26-49$ years & $32(43.8)$ & $33(45.8)$ & $65(44.8)$ & \\
\hline $50+$ years & $1(1.4)$ & $15(20.8)$ & $16(11.0)$ & \\
\hline \multicolumn{5}{|l|}{ Race/ethnicity, $n$ (\%) } \\
\hline White & $55(75.3)$ & $60(83.3)$ & $115(79.3)$ & \multirow[t]{6}{*}{0.235} \\
\hline Black/African American & $5(6.8)$ & $1(1.4)$ & $6.0(4.1)$ & \\
\hline Latinx/Hispanic & $2(2.7)$ & $3(4.2)$ & $5.0(3.4)$ & \\
\hline Multiracial & $7(9.6)$ & $5(6.9)$ & $12.0(8.3)$ & \\
\hline Other & $3(4.1)$ & $2(2.8)$ & $5.0(3.4)$ & \\
\hline Not indicated & $1(1.4)$ & $1(1.4)$ & $2.0(1.4)$ & \\
\hline \multicolumn{5}{|l|}{ Employment, $n(\%)$} \\
\hline Working full or part time & 51 (69.9) & $48(66.7)$ & $99(68.3)$ & \multirow[t]{3}{*}{0.200} \\
\hline $\begin{array}{l}\text { Not working (unemployed, } \\
\text { retired, or disabled) }\end{array}$ & $10(13.7)$ & $17(23.6)$ & 27 (18.6) & \\
\hline Student & $12(16.4)$ & $7(9.7)$ & $19(13.1)$ & \\
\hline \multicolumn{4}{|l|}{ Income, $n$ (\%) } & \multirow[t]{6}{*}{0.587} \\
\hline At or below poverty level & $29(39.7)$ & $31(43.1)$ & $60(41.4)$ & \\
\hline $100-200 \%$ of poverty level & $8(11.0)$ & $11(15.3)$ & $19(13.1)$ & \\
\hline $200-300 \%$ of poverty level & $17(23.3)$ & $11(15.3)$ & $28(19.3)$ & \\
\hline$>300 \%$ of poverty level & $17(23.3)$ & $19(26.4)$ & $36(24.8)$ & \\
\hline Not indicated & $2(2.7)$ & $0(0.0)$ & $2(1.4)$ & \\
\hline
\end{tabular}

Bold indicates statistical significance $(p<0.05)$. Reported $p$-values are from Fisher's exact tests wherein cell sizes are small $(<5)$. Response rate was $100 \%$ for all variables except where table states "Not indicated."

$\mathrm{SD}$, standard deviation; TF, trans feminine; TM, trans masculine.

gender identity-related characteristics, (3) sexual history, and (4) mental health. Presence or absence of these characteristics was determined from the EHR for the 5 -year study period through a combination of automated queries and manual chart audit. The statistical predictors are detailed in Tables 1-4.

\section{Statistical analysis}

Univariate statistics were used to examine the distributions of all variables (mean, median, standard deviation, frequency, and proportion) overall and stratified by gender identity: trans masculine (TM) versus trans feminine (TF). Following descriptive statistics, bivariate analyses were conducted by gender identity to compare TM versus TF patients. Mann-Whitney $U$ tests were used to assess median differences for non-normally distributed continuous variables (i.e., "current age" and "age of medical gender affirmation"). Pearson's chi-square $\left(\chi^{2}\right)$ tests with Yates' correction were used to examine any differences in expected and observed proportions by gender identity. Where sparse data caused expected counts to be $<5$, Fisher's exact tests were utilized to obtain exact $p$-values to accompany $\chi^{2}$ test statistics.

Multivariable regression analyses were conducted with variables that had $>85 \%$ completeness and were selected based on clinical hypotheses. Among patients in the sample, $56(27.9 \%)$ did not have complete data for all desired variables and were excluded from the multivariable regression procedures. Thus, multivariable regression analyses were restricted to 145 patients (72.1\% of the original sample: a random distribution of $73 \mathrm{TM}$ and 72 TF patients). To assess how sample characteristics of these 145 eligible participants compared with the health center's overall pool of 1683 transgender patients, we conducted $\chi^{2}$ analyses comparing the 2 groups across each race/ethnicity category and found no statistically significant differences at the $p<0.05$ level in the proportion of patients who identified as "white," "black/African American," "Latinx/Hispanic," "multiracial," "other," or "not indicated." A "dummy" variable coding exclusion versus inclusion (missing vs. not missing) in the multivariable analyses was created and analyzed as a bivariate against each regression outcome to ascertain whether exclusion introduced bias. In these sensitivity analyses, this covariate did not reach statistical significance in any of the models.

Table 2. Gender Identity-Related Characteristics

\begin{tabular}{|c|c|c|c|c|}
\hline Variable & $\mathrm{TM}(n=73), n(\%)$ & TF $(n=72), n(\%)$ & Total $(N=145), n(\%)$ & TM vs. TF, $p$ \\
\hline Gender identity when established care at community health center & & & & $<0.001$ \\
\hline Female & $5(6.8)$ & $49(68.1)$ & $54(37.2)$ & \\
\hline Male & $49(67.1)$ & $7(9.7)$ & $56(38.6)$ & \\
\hline Genderqueer/nonbinary & $19(26.0)$ & $16(22.2)$ & $35(24.1)$ & \\
\hline Hormones prescribed by primary care provider & $69(94.5)$ & $69(95.8)$ & $138(95.2)$ & 1.000 \\
\hline Current medically unmonitored hormone use & $0(0.0)$ & $3(4.2)$ & $3(2.1)$ & 0.120 \\
\hline Any past medically unmonitored hormone use & $0(0.0)$ & $6(8.3)$ & $6(4.1)$ & 0.013 \\
\hline Any gender-affirming surgery & $27(37.0)$ & $20(27.8)$ & $47(32.4)$ & 0.314 \\
\hline \multicolumn{5}{|l|}{ Age of hormone therapy initiation } \\
\hline Mean (SD) & $27.9(7.1)$ & $33.3(13.2)$ & $31.8(11.1)$ & \\
\hline Median & 24.0 & 27.0 & 27.0 & 0.001 \\
\hline Range & $8-50$ & $15-64$ & $8-64$ & \\
\hline
\end{tabular}

Bold indicates statistical significance $(p<0.05)$. Reported $p$-values are from Fisher's exact tests wherein cell sizes are small $(<5)$. Response rate was $100 \%$ for all variables except where table states "Not indicated." 
Table 3. Sexual Orientation and History

\begin{tabular}{|c|c|c|c|c|}
\hline Variable & $\begin{array}{c}\text { TM } \\
(n=73) \\
n(\%)\end{array}$ & $\begin{array}{c}\mathrm{TF} \\
(n=72) \\
n(\%)\end{array}$ & $\begin{array}{c}\text { Total } \\
(N=145), \\
n(\%)\end{array}$ & $\begin{array}{c}\text { TM vs. } \\
\text { TF, } p\end{array}$ \\
\hline Sexual orientation & & & & 0.019 \\
\hline Bisexual & $9(12.3)$ & $22(30.6)$ & $31(21.4)$ & \\
\hline Lesbian, gay, or homosexual & $14(19.2)$ & $15(20.8)$ & $29(20.0)$ & \\
\hline Straight or heterosexual & $15(20.5)$ & $9(12.5)$ & $24(16.6)$ & \\
\hline Something else & $31(42.5)$ & $18(25.0)$ & $49(33.8)$ & \\
\hline Does not know & $4(5.5)$ & $8(11.1)$ & $12(8.3)$ & \\
\hline Primary sex partner & $48(65.8)$ & $42(58.3)$ & $90(62.1)$ & 0.454 \\
\hline Casual sex partner(s) & $5(6.8)$ & $5(6.9)$ & $10(6.9)$ & 0.982 \\
\hline Any STI diagnosis & $13(17.8)$ & $7(9.7)$ & $20(13.8)$ & 0.242 \\
\hline
\end{tabular}

Bold indicates statistical significance $(p<0.05)$. Reported $p$-values are from Fisher's exact tests wherein cell sizes are small $(<5)$. Response rate was $100 \%$ for all variables except where table states "Not indicated."

To increase power, the sample was analyzed in aggregate and not stratified by gender identity. Gender identity was included as a covariate. Model building initially focused on examining bivariate models for each of the variables listed in Tables 1-4 to identify those with statistical significance. Factors significant at the $p<0.05$ level were entered into a multivariable model, and variable selection for independent associations was implemented using the backward elimination method. All significant variables were entered into the equation and the least useful variables were eliminated one at a time using the smallest $\chi^{2}$ to remove, to a threshold for inclusion of $p<0.05$. $^{28}$
For the binary outcome variable, multivariable logistic regression models were fit to examine factors associated with history of gender-affirming surgery. For the regression on history of gender-affirming surgery, the variable "current age" was a significant bivariate due to its strong correlation with a number of other variables and was, therefore, entered after conducting model selection with the other variables. The inference for the final model for history of gender-affirming surgery did not differ from the model before adding the "current age" variable. The "current age" variable was excluded from the regression on "age of hormone therapy initiation" due to its especially strong correlation in that case.

Table 4. Mental Health

\begin{tabular}{|c|c|c|c|c|}
\hline Variable & $\mathrm{TM}(n=73), n(\%)$ & TF $(n=72), n(\%)$ & Total $(N=145), n(\%)$ & TM vs. TF, $p$ \\
\hline Lifetime substance use & $59(80.8)$ & $54(75.0)$ & $113(77.9)$ & 0.519 \\
\hline Current alcohol use & $52(71.2)$ & $49(68.1)$ & $101(69.7)$ & 0.814 \\
\hline Past alcohol use & $7(9.6)$ & $6(8.3)$ & $13(9.0)$ & 0.791 \\
\hline Current cannabis use & $26(35.6)$ & $22(30.6)$ & $48(33.1)$ & 0.638 \\
\hline Any assessed psychiatric diagnoses & 45 (61.6) & $38(52.8)$ & $83(57.2)$ & 0.362 \\
\hline Lifetime substance use disorder & $13(17.8)$ & $17(23.6)$ & $30(20.7)$ & 0.511 \\
\hline Current alcohol use disorder & $3(4.1)$ & $8(11.1)$ & $11(7.6)$ & 0.129 \\
\hline Past alcohol use disorder & $5(6.8)$ & $5(6.9)$ & $10(6.9)$ & 0.982 \\
\hline Current cannabis use disorder & $7(9.6)$ & $5(6.9)$ & $12(8.3)$ & 0.782 \\
\hline PTSD & $6(8.2)$ & $3(4.2)$ & $9(6.2)$ & 0.494 \\
\hline Anxiety disorder & $28(38.4)$ & $13(18.1)$ & $41(28.3)$ & 0.011 \\
\hline Major depressive disorder & $30(41.1)$ & $25(34.7)$ & 55 (37.9) & 0.536 \\
\hline Bipolar disorder & $2(2.7)$ & $2(2.8)$ & $4(2.8)$ & 1.000 \\
\hline Personality disorder & $2(2.7)$ & $1(1.4)$ & $3(2.1)$ & 1.000 \\
\hline History of suicide attempt & $11(15.1)$ & $9(12.5)$ & $20(13.8)$ & 0.836 \\
\hline History of inpatient psychiatric treatment & $11(15.1)$ & $6(8.3)$ & $17(11.7)$ & 0.316 \\
\hline History of residential or partial hospitalization program & $2(2.7)$ & $3(4.2)$ & $5(3.4)$ & 0.681 \\
\hline Current psychotherapist & $47(64.4)$ & $42(58.3)$ & $89(61.4)$ & 0.564 \\
\hline Current psychopharmacologist & $36(49.3)$ & $29(40.3)$ & $65(44.8)$ & 0.354 \\
\hline Psychiatrist integrated with primary care & $3(4.1)$ & $3(4.2)$ & $6(4.1)$ & 1.000 \\
\hline Addictions program integrated with primary care & $0(0.0)$ & $2(2.8)$ & $2(1.4)$ & 0.245 \\
\hline Psychiatrist elsewhere & $22(30.1)$ & $13(18.1)$ & $35(24.1)$ & 0.132 \\
\hline Current case management utilization & $18(24.7)$ & $15(20.8)$ & $33(22.8)$ & 0.723 \\
\hline
\end{tabular}

Bold indicates statistical significance $(p<0.05)$. Reported $p$-values are from Fisher's exact tests wherein cell sizes are small $(<5)$. Response rate was $100 \%$ for all variables except where table states "Not indicated." 
For the continuous outcome variable "age of hormone therapy initiation," given its distribution and dispersion, negative binomial multivariable regression models were fit to examine factors associated with this outcome variable. Data analysis was conducted using SAS Studio, Release 3.5, and Microsoft Excel 2010.

\section{Results}

Descriptive and bivariate analyses

Characteristics of the study sample are presented in Tables 1-4 for TM and TF participants separately, and for the total sample in aggregate. Also included in these tables are bivariate statistics comparing TM versus TF individuals.

Demographics. Overall, $50 \%$ of the sample was TM participants and 50\% TF participants. The mean age of participants was $31.8(\mathrm{SD}=11.5)$ years. The difference in median age between TM participants ( 25 years old) and TF participants (30 years old) was statistically significant (Mann-Whitney $U=1753, p=0.001$ ). Four percent of the sample identified as African American/black, $3 \%$ identified as Latinx/Hispanic, and $8 \%$ identified as multiracial. Forty-one percent of the sample reported living at or below the federal poverty level (Table 1).

Gender identity-related characteristics. Nearly one in four (24.1\%) patients identified as genderqueer/nonbinary, in roughly equal proportions for TM (26.0\%) and TF (22.2\%). Within the study sample, $95 \%$ of participants were actively prescribed gender-affirming hormones by their primary care provider. The mean age of hormone therapy initiation was $31.8(\mathrm{SD}=11.1)$ years. The difference in median age of hormone therapy initiation between TM participants (24 years old) and TF participants (27 years old) was statistically significant (Mann-Whitney $U=1802.5, p=0.001)$. TF participants were more likely than TM participants to have a history of medically unmonitored hormone use, and this difference was statistically significant, $\chi^{2}(2, N=145)=4.42, p<0.013$ (Table 2$)$.

Thirty-two percent of participants had a history of gender-affirming surgery. Among TM participants, $37 \%$ had a history of gender-affirming surgery, with $35.6 \%$ undergoing chest construction, 5.5\% undergoing hysterectomy, and 5.5\% undergoing oophorectomy. In addition, 1 TM patient in the original sample of 201 adults had undergone phalloplasty and was excluded from multivariable regression procedures due to not having complete data for all desired variables. Among $\mathrm{TF}, 27.8 \%$ had a history of gender-affirming procedures, with $8.3 \%$ undergoing breast augmentation, $6.9 \%$ undergoing vaginoplasty, $2.8 \%$ undergoing facial feminization, $2.8 \%$ undergoing orchiectomy without vaginoplasty, and $12.5 \%$ undergoing other gender-affirming procedures (i.e., other implants or electrolysis).

Sexual history. The most commonly reported sexual orientation was "something else" (33.8\%). Substantial heterogeneity in the distribution of sexual orientation was found by gender identity, $\chi^{2}(4, N=145)=11.76$, $p=0.019$, with a higher proportion of TM versus TF endorsing "something else" ( $42.5 \%$ vs. $25.0 \%)$ or "straight" (20.5\% vs. $12.5 \%$ ), and a higher proportion of TF versus TM identifying as "bisexual" (30.6\% vs. $12.3 \%)$ or "does not know" (11.1\% vs. $5.5 \%)$. The majority of the sample (62.1\%) had a primary sex partner, $6.9 \%$ reported one or more casual sex partners, and $13.8 \%$ had a sexually transmitted infection diagnosis history (Table 3).

Mental health. Substance use disorders and other psychiatric diagnoses were high in the sample, with more than half (57.2\%) diagnosed with a psychiatric disorder. TM participants were more likely than TF participants to have an anxiety disorder, and this difference was statistically significant, $\chi^{2}(1, N=145)=6.40$, $p<0.011$ (Table 4).

\section{Multivariable regression models}

Table 5 presents multivariable logistic regression modeling with history of any gender-affirming surgery as the primary outcome. Factors associated with a statistically significant increase in the odds of undergoing gender-affirming surgery (at $p<0.05)$ were $(1)$ age in years, (2) income $>300 \%$ of the federal poverty level,

Table 5. Significant Outcomes of Binary Logistic Regressions on History of Any Gender-Affirming Surgery

\begin{tabular}{|c|c|c|c|c|}
\hline \multirow[b]{2}{*}{ Variable } & \multicolumn{2}{|c|}{ Bivariate models } & \multicolumn{2}{|c|}{ Multivariable model } \\
\hline & OR $(95 \% \mathrm{Cl})$ & $p$ & aOR $(95 \% \mathrm{Cl})$ & $p$ \\
\hline \multicolumn{5}{|l|}{ Demographics } \\
\hline Age in years & $1.04(1.01-1.07)$ & 0.021 & $1.03(1.00-1.07)$ & 0.047 \\
\hline $\begin{array}{c}\text { Income at } 100-200 \% \\
\text { of poverty level }\end{array}$ & $0.21(0.05-0.96)$ & 0.044 & & \\
\hline $\begin{array}{l}\text { Income }>300 \% \\
\text { of poverty level }\end{array}$ & $2.76(1.27-6.01)$ & 0.011 & $3.17(1.34-7.52)$ & 0.009 \\
\hline \multicolumn{5}{|l|}{ Sexual history } \\
\hline Bisexual & $0.33(0.12-0.92)$ & 0.035 & $0.23(0.07-0.71)$ & 0.011 \\
\hline Straight/heterosexual & $2.46(1.01-5.99)$ & 0.048 & & \\
\hline \multicolumn{5}{|l|}{ Mental health } \\
\hline $\begin{array}{l}\text { Current } \\
\text { psychotherapist }\end{array}$ & $0.41(0.20-0.83)$ & 0.014 & $0.35(0.16-0.76)$ & 0.008 \\
\hline
\end{tabular}

$N=145$. Bold indicates statistical significance $(p<0.05)$. aOR, adjusted odds ratio; $95 \% \mathrm{Cl}, 95 \%$ confidence interval; OR, odds ratio. 
Table 6. Significant Outcomes of Negative Binomial Regressions on Age of Hormone Therapy Initiation

\begin{tabular}{|c|c|c|c|c|}
\hline \multirow[b]{2}{*}{ Variable } & \multicolumn{2}{|c|}{ Bivariate models } & \multicolumn{2}{|c|}{ Multivariable model } \\
\hline & Coefficient $(95 \% \mathrm{Cl})$ & $p$ & Coefficient $(95 \% \mathrm{Cl})$ & $p$ \\
\hline \multicolumn{5}{|l|}{ Demographics } \\
\hline Male sex assigned at birth (i.e., TF) & $0.24(0.13-0.34)$ & $<0.001$ & $0.21(0.11-0.31)$ & $<0.001$ \\
\hline Student status & $-0.21(-0.38$ to -0.04$)$ & 0.013 & $-0.16(-0.31$ to -0.01$)$ & 0.041 \\
\hline Income $>300 \%$ of poverty level & $0.14(0.01-0.27)$ & 0.030 & & \\
\hline \multicolumn{5}{|l|}{ Gender identity-related characteristics } \\
\hline Male gender identity & $-0.17(-0.29$ to -0.06$)$ & 0.002 & & \\
\hline \multicolumn{5}{|l|}{ Sexual history } \\
\hline Straight/heterosexual & $-0.17(-0.33$ to -0.02$)$ & 0.024 & $-0.15(-0.28$ to -0.01$)$ & 0.032 \\
\hline Casual sex partner(s) & $-0.28(-0.50$ to -0.05$)$ & 0.017 & $-0.31(-0.51$ to -0.10$)$ & 0.003 \\
\hline \multicolumn{5}{|l|}{ Mental health } \\
\hline Past alcohol use & $0.20(0.01-0.39)$ & 0.035 & & \\
\hline Lifetime substance use disorder & $0.15(0.02-0.29)$ & 0.026 & & \\
\hline Current alcohol use disorder & $0.22(0.02-0.43)$ & 0.031 & & \\
\hline Past alcohol use disorder & $0.30(0.09-0.51)$ & 0.005 & $0.27(0.08-0.45)$ & 0.005 \\
\hline
\end{tabular}

$N=145$. Bold indicates statistical significance $(p<0.05)$.

(3) not identifying as bisexual, and (4) not having a current psychotherapist.

For the multivariable negative binomial regression model given in Table 6 , the primary outcome is age of initiation of gender-affirming hormone therapy. There was a statistically significant association (at $p<0.05$ ) between younger age at time of hormone therapy initiation and each of the following factors: (1) being TM, (2) being a student, (3) identifying as straight/heterosexual, (4) having casual sex partner(s), and (5) having no past alcohol use disorder.

\section{Discussion}

In this EHR review study based at an LGBT-focused urban health center, almost all transgender adults were receiving gender-affirming hormone therapy from their primary care provider, similar to the high prevalence of prescribed hormone therapy in other samples of transgender adults from specialized clinical settings. ${ }^{29-31}$ Compared with TM participants, we found that TF participants were first prescribed gender-affirming hormone therapy at an older age and also had a greater likelihood of past medically unmonitored hormone use. These differences may be related to TF participants being older than TM participants in the sample (e.g., age cohort effects) or to higher prevalence of discriminatory experiences and/or more barriers to accessing gender-affirming health services among TF people. ${ }^{32}$ Our findings are consistent with previous reports indicating that medically unmonitored hormone use among TF people typically occurs in the context of significant barriers to accessing and receiving gender-affirming medical care. ${ }^{15,18,33,34}$ The results in this study extend findings from previous research with TF people that showed prevalence of medically unmonitored hormone use as high as $60 \%$ in the United States and Canada. ${ }^{13,15,34,35}$

Within our study sample, $32 \%$ of participants had a documented history of gender-affirming surgery. This finding is similar to a recent retrospective chart review conducted at a specialized endocrinology clinic where $35 \%$ of transgender patients had a history of genderaffirming surgery. ${ }^{36}$ Consistent with our study, this chart review found that TM patients underwent chest construction surgery more often than hysterectomy and/or oophorectomy, and that TF patients underwent either breast augmentation or genital surgery more often than facial feminization surgery. A recent survey study of both TM and TF adults found that $23 \%$ of respondents reported past chest surgery and $11 \%$ reported past genital reconstruction surgery. ${ }^{22}$ In contrast to that study, our analysis did not exclude the large subgroup of participants (24.1\%) with nonbinary gender identities (e.g., genderqueer), who often face unique barriers to receiving gender-affirming surgeries in the context of surgical prerequisites that may favor candidates with more traditional binary gender identities. $^{2}$ To our knowledge, no studies to date have assessed whether nonbinary transgender adults are as likely to seek gender-affirming surgeries as their counterparts with binary gender identities.

We found that transgender adults with a history of gender-affirming surgery were more likely to be older and to have a higher income than those without a history of gender-affirming surgery. Based on EHR data, we were unable to consistently assess age at time of 
first gender-affirming surgery and whether this age was associated with prior duration of engagement in gender-affirming hormone therapy. Older transgender adults are developmentally more likely to have established the financial security, psychosocial stability, and support networks to facilitate effectively accessing gender-affirming surgical care, even in the context of recent expansion of insurance coverage for genderaffirming surgical procedures in the United States. ${ }^{19,37}$ It is also possible that transgender people who undergo gender-affirming surgery may be more capable of increasing their incomes because of the higher quality of life and improved functioning observed among transgender adults after accessing gender-affirming care. ${ }^{4}$ In the context of prior research showing that transgender adults in the United States on the low end of the socioeconomic status (SES) spectrum (e.g., low income) report higher rates of lifetime refusal of healthcare than those of higher SES, ${ }^{38}$ our findings point to an SES gradient in access to gender-affirming surgeries, with disparities in access for low-income transgender adults.

Bisexual-identified participants in this sample were less likely to have had gender-affirming surgery. Some experts on the topic of sexuality among transgender people have pointed to the adverse impact of medical gatekeeping by clinicians who historically viewed a "non-normative" sexual orientation (e.g., not "straight" or "heterosexual") as an ineligibility criterion for gender-affirming body modifications. ${ }^{39}$ The EHR data did not afford us the opportunity to assess differences based on sexual orientation in the likelihood of seeking gender-affirming surgery. Not currently having a psychotherapist was associated with a history of genderaffirming surgery. The lower likelihood of having a psychotherapist among participants with past genderaffirming surgery may be related to the high degree of psychiatric stability typically required to access gender-affirming surgical procedures, ${ }^{2}$ consistent with previous research indicating that the presence of a mood disorder is associated with a longer time lag between initially presenting for gender-affirming outpatient care and ultimately accessing gender-affirming genital surgery. ${ }^{40}$ Alternatively, this result may be due to significant improvement in psychological functioning among transgender people whose gender has been affirmed through access to healthcare. ${ }^{4}$

This study also assessed age of initiation of genderaffirming hormone prescriptions. Transgender adults who accessed hormone prescriptions early in life were more likely to be TM and to be a student. It may be that transgender participants who accessed hormone prescriptions at a younger age were more likely to be TM in light of greater stigma and barriers to healthcare linkage experienced by TF people. ${ }^{32}$ Student status among transgender adults may be a marker of higher educational and economic status associated with greater health literacy and financial resources, which could facilitate earlier access to hormone prescriptions. Another possibility is that access to genderaffirming hormone prescriptions early in life led to enhanced psychological functioning and, therefore, more advanced subsequent educational attainment. ${ }^{4}$

With regard to sexual history, accessing hormone prescriptions at younger ages was associated with identifying as straight/heterosexual and having casual sex partner(s). Transgender participants with societally defined normative sexual orientations (e.g., "straight" or "heterosexual" people) may have encountered less medical gatekeeping when seeking hormone prescriptions early in life from clinicians who viewed their more traditional sexuality as an indicator of the appropriateness of their transgender identity. ${ }^{39}$ It is also possible that transgender adults who are not heterosexual- or straight-identified tend to seek hormone therapy later in life or with lower prevalence, or that among transgender adults this subpopulation encounters more barriers to healthcare access in general. Accessing gender-affirming hormone prescriptions early in life may have been associated with having one or more casual sex partners due to greater comfort engaging in sexual activity among transgender adults after accessing medical gender affirmation. ${ }^{41-43}$

In terms of mental health, younger age of hormone prescription access was associated with not having a past alcohol use disorder. Participants who accessed gender-affirming hormones at a younger age may have had less of the behavioral disorganization or clinician-level gatekeeping that can occur in the context of an alcohol use disorder and impede linkage to gender-affirming medical care. Alternatively, transgender adults with access to gender-affirming hormones at an earlier age may have subsequently experienced less gender dysphoria and, therefore, been less likely to cope with gender-related distress by means of at-risk alcohol use. ${ }^{2,9,32}$ That younger age of hormone therapy initiation was protective for alcohol use disorder suggests that early access to gender-affirming hormones may buffer against subsequent adverse substance use disorder outcomes. 
A limitation of this study is its cross-sectional design, which does not permit causal inference about the relationships of independent variables to past genderaffirming surgery or age of hormone therapy initiation. Another limitation of our work is the derivation of this study sample of transgender adults from one LGBTspecialized urban community health center in the United States with a primarily white patient population, which reduces our ability to generalize these findings to all transgender communities in any region. The retrospective EHR review method is susceptible to unintended bias related to documentation that may be incomplete, clinical information that may not have been recorded, and subjective interpretation of some variables of interest from the charts (e.g., defining "casual" and "primary" sex partners). ${ }^{26,27,44-46}$ Individual variability among members of the clinical care team in their practice of entering information into EHR fields may have also diminished data integrity. ${ }^{47}$ Finally, stigma was not assessed as part of this study because this information is not captured in EHRs. Future research would benefit from considering the role of stigma in access to and receipt of gender-affirming hormones and surgical interventions. ${ }^{48}$

\section{Conclusion}

Although not without limitations, this study extends our understanding of the prevalence of genderaffirming surgeries and age of hormone therapy initiation among both TM and TF adults. Our findings suggest that accessing these gender-affirming treatments is associated with better mental health, higher socioeconomic status, and having a heterosexual orientation. To our knowledge, this is the first study to comprehensively examine the relationship of these two categories of gender-affirming clinical care with demographics, gender identity, sexual history, and mental health. An additional strength of our research is that approximately one-quarter of the study sample were genderqueer/nonbinary patients at the time of clinical care initiation at the health center, offering greater heterogeneity of gender identities in the sample and reflecting the reality of clinical practices serving transgender patients. Our study also serves as a demonstration of how systematic gender identity data collection in EHRs provides opportunities to better understand the unique health needs of transgender people engaged in clinical care. Future studies ought to continue to focus on barriers and facilitators of gender-affirming care for transgender adults, to facilitate the development of individual- and systems-level interventions, as well as policies, that help expand access to medically necessary care for this highly underserved and vulnerable population, reduce health disparities, and improve both physical and mental health outcomes.

\section{Acknowledgments}

This research was supported by Grant U30CS22742 from the Health Resources and Services Administration Bureau of Primary Health Care and by Grant R34MH104072 from the National Institute of Mental Health.

\section{Author Disclosure Statement}

No competing financial interests exist.

\section{References}

1. Stroumsa D. The state of transgender health care: policy, law, and medical frameworks. Am J Public Health. 2014;104:e31-e38.

2. Coleman $E$, Bockting W, Botzer M, et al. Standards of care for the health of transsexual, transgender, and gender-nonconforming people, version 7. Int J Transgend. 2012;13:165-232.

3. American Medical Association House of Delegates. H-185.950 Removing Financial Barriers to Care for Transgender Patients. American Medical Association: Chicago, IL, 2008.

4. White Hughto JM, Reisner SL. A systematic review of the effects of hormone therapy on psychological functioning and quality of life in transgender individuals. Transgend Health. 2016;1:21-31.

5. Lindqvist EK, Sigurjonsson $\mathrm{H}$, Mollermark $\mathrm{C}$, et al. Quality of life improves early after gender reassignment surgery in transgender women. Eur J Plast Surg. 2017;40:223-226.

6. Papadopulos NA, Lelle JD, Zavlin D, et al. Quality of life and patient satisfaction following male-to-female sex reassignment surgery. J Sex Med. 2017;14:721-730.

7. Keuroghlian AS, Reisner SL, White JM, Weiss RD. Substance use and treatment of substance use disorders in a community sample of transgender adults. Drug Alcohol Depend. 2015;152:139-146.

8. Wilson EC, Chen YH, Arayasirikul S, et al. Connecting the dots: examining transgender women's utilization of transition-related medical care and associations with mental health, substance use, and HIV. J Urban Health. 2015;92:182-192.

9. James SE, Herman JL, Rankin S, et al. The Report of the 2015 U.S. Transgender Survey. Washington, D.C.: National Center for Transgender Equality, 2016

10. Safer JD, Coleman E, Feldman J, et al. Barriers to healthcare for transgender individuals. Curr Opin Endocrinol Diabetes Obes. 2016;23:168171.

11. Keuroghlian AS, Ard KL, Makadon HJ. Advancing health equity for lesbian, gay, bisexual and transgender (LGBT) people through sexual health education and LGBT-affirming health care environments. Sex Health 2017. [Epub ahead of print]; DOI: 10.1071/SH16145.

12. Xavier J. Final Report of the Washington Transgender Needs Assessment Survey. Washington, DC: Administration for HIV and AIDS, Government of the District of Columbia, 2000

13. Garofalo R, Deleon J, Osmer E, et al. Overlooked, misunderstood and at-risk: exploring the lives and HIV risk of ethnic minority male-to-female transgender youth. J Adolesc Health. 2006;38:230-236.

14. Benotsch EG, Zimmerman R, Cathers $L$, et al. Non-medical use of prescription drugs, polysubstance use, and mental health in transgender adults. Drug Alcohol Depend. 2013;132:391-394.

15. Rotondi NK, Bauer GR, Scanlon K, et al. Nonprescribed hormone use and self-performed surgeries: "do-it-yourself" transitions in transgender communities in Ontario, Canada. Am J Public Health. 2013;103:1830-1836.

16. Operario D, Nemoto T. HIV in transgender communities: syndemic dynamics and a need for multicomponent interventions. J Acquir Immune Defic Syndr. 2010;55(Suppl 2):S91-S93. 
17. Aguayo-Romero RA, Reisen CA, Zea MC, et al. Gender affirmation and body modification among transgender persons in Bogota, Colombia. Int J Transgend. 2015;16:103-115.

18. Radix A, Sevelius J, Deutsch MB. Transgender women, hormonal therapy and HIV treatment: a comprehensive review of the literature and recommendations for best practices. J Int AIDS Soc. 2016;19(Suppl 2):20810.

19. Berli JU, Knudson G, Fraser L, et al. What surgeons need to know about gender confirmation surgery when providing care for transgender individuals: a review. JAMA Surg. 2017;152:394-400.

20. Beek TF, Kreukels BP, Cohen-Kettenis PT, Steensma TD. Partial treatment requests and underlying motives of applicants for gender affirming interventions. J Sex Med. 2015;12:2201-2205.

21. Giami A, Beaubatie E. Gender identification and sex reassignment surgery in the trans population: a survey study in France. Arch Sex Behav. 2014;43:1491-1501.

22. Sineath RC, Woodyatt C, Sanchez T, et al. Determinants of and barriers to hormonal and surgical treatment receipt among transgender people. Transgend Health. 2016;1:129-136.

23. Reisner SL, Bradford J, Hopwood R, et al. Comprehensive transgender healthcare: the gender affirming clinical and public health model of Fenway Health. J Urban Health. 2015;92:584-592.

24. Mayer K, Appelbaum J, Rogers T, et al. The evolution of the Fenway Community Health model. Am J Public Health. 2001;91:892-894.

25. Thompson SK. Simple Random sampling. In: Sampling, 3rd ed. Hoboken: John Wiley \& Sons, 2012, pp. 9-37.

26. Myers L, Stevens J. Using EHR to conduct outcome and health services research. In: Secondary Analysis of Electronic Health Records. (MIT Critical Data; ed.). Cham, Switzerland: Springer International Publishing, 2016, pp. 61-70.

27. Casey JA, Schwartz BS, Stewart WF, Adler NE. Using electronic health records for population health research: a review of methods and applications. Annu Rev Public Health. 2016;37:61-81.

28. Afifi A, May S, Clark VA. Computer-Aided Multivariate Analysis, 4th ed. Boca Raton: Chapman and Hall/CRC, 2004

29. Gorin-Lazard A, Baumstarck K, Boyer L, et al. Hormonal therapy is associated with better self-esteem, mood, and quality of life in transsexuals. J Nerv Ment Dis. 2013;201:996-1000.

30. Judge C, O'Donovan C, Callaghan G, et al. Gender dysphoria-prevalence and co-morbidities in an Irish adult population. Front Endocrinol (Lausanne). 2014;5:87.

31. Wierckx K, Elaut E, Declercq E, et al. Prevalence of cardiovascular disease and cancer during cross-sex hormone therapy in a large cohort of trans persons: a case-control study. Eur J Endocrinol. 2013;169:471-478.

32. Grant JM, Mottet LA, Tanis J, et al. Injustice at Every Turn: A Report of the National Transgender Discrimination Survey. Washington, D.C.: National Center for Transgender Equality, and the National Gay and Lesbian Task Force, 2011.

33. Mepham N, Bouman WP, Arcelus J, et al. People with gender dysphoria who self-prescribe cross-sex hormones: prevalence, sources, and side effects knowledge. J Sex Med. 2014;11:2995-3001.

34. Sanchez NF, Sanchez JP, Danoff A. Health care utilization, barriers to care, and hormone usage among male-to-female transgender persons in New York City. Am J Public Health. 2009;99:713-719.

35. de Haan G, Santos GM, Arayasirikul S, Raymond HF. Non-prescribed hormone use and barriers to care for transgender women in San Francisco. LGBT Health. 2015;2:313-323.

36. Kailas M, Lu HMS, Rothman EF, Safer JD. Prevalence and types of genderaffirming surgery among a sample of transgender endocrinology patients prior to state expansion of insurance coverage. Endocr Pract. 2017;23:780-786.

37. Baker KE. The future of transgender coverage. N Engl J Med. 2017;376:1801-1804.
38. White Hughto JM, Murchison GR, Clark K, et al. Geographic and individual differences in healthcare access for U.S. transgender adults: a multilevel analysis. LGBT Health. 2016;3:424-433.

39. Rowniak S, Chesla C. Coming out for a third time: transmen, sexual orientation, and identity. Arch Sex Behav. 2013;42:449-461.

40. Leinung M, Urizar M, Patel N, Sood S. Endocrine treatment of transsexual persons: extensive personal experience. Endocr Pract. 2013;19:644-650.

41. Doorduin T, van Berlo W. Trans people's experience of sexuality in the Netherlands: a pilot study. J Homosex. 2014;61:654-672.

42. Bauer GR, Hammond R. Toward a broader conceptualization of trans women's sexual health. Can J Hum Sex. 2015;24:1-11.

43. Hill-Meyer T, Scarborough D. Sexuality. In: Trans Bodies, Trans Selves: A Resource for the Transgender Community. (Erickson-Schroth L; ed) New York: Oxford University Press, 2014, pp. 355-389.

44. Dworkin RJ. Hidden bias in the use of archival data. Eval Health Prof 1987;10:173-185.

45. Gearing RE, Mian IA, Barber J, Ickowicz A. A methodology for conducting retrospective chart review research in child and adolescent psychiatry. J Can Acad Child Adolesc Psychiatry. 2006;15:126-134.

46. Hess DR. Retrospective studies and chart reviews. Respir Care. 2004;49:1171-1174.

47. Pan L, Fergusson D, Schweitzer I, Hebert PC. Ensuring high accuracy of data abstracted from patient charts: the use of a standardized medical record as a training tool. J Clin Epidemiol. 2005;58:918-923.

48. White Hughto JM, Reisner SL, Pachankis JE. Transgender stigma and health: a critical review of stigma determinants, mechanisms, and interventions. Soc Sci Med. 2015;147:222-231.

Cite this article as: Beckwith $\mathrm{N}$, Reisner $\mathrm{SL}$, Zaslow S, Mayer $\mathrm{KH}$, Keuroghlian AS (2017) Factors associated with gender-affirming surgery and age of hormone therapy initiation among transgender adults, Transgender Health 2:1, 156-164, DOI: 10.1089/trgh.2017.0028.

$\begin{aligned} & \text { Abbreviations Used } \\ & \mathrm{aOR}=\text { adjusted odds ratio } \\ & 95 \% \mathrm{Cl}=95 \% \text { confidence interval } \\ & \mathrm{EHR}=\text { electronic health record } \\ & \mathrm{OR}=\text { odds ratio } \\ & \mathrm{SD}=\text { standard deviation } \\ & \mathrm{SES}=\text { socioeconomic status } \\ & \mathrm{TF}=\text { trans feminine } \\ & \mathrm{TM}=\text { trans masculine }\end{aligned}$

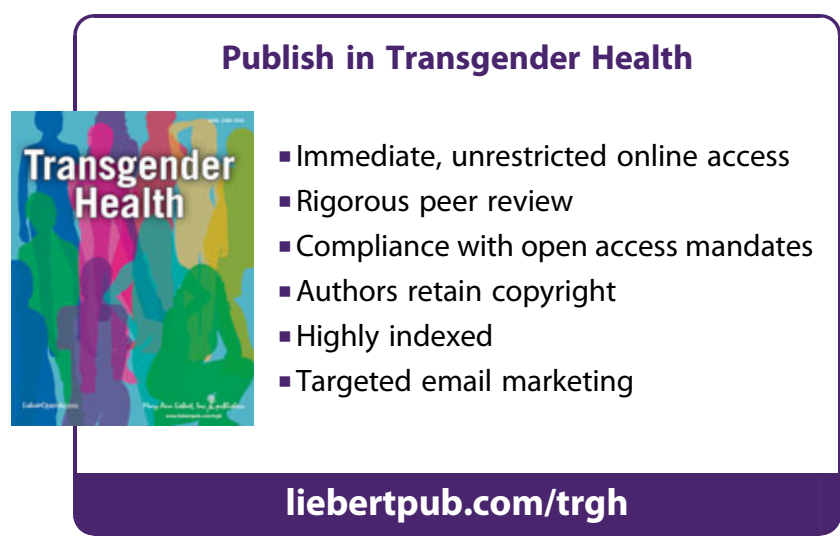

